\title{
Differential effects of omitting comparison stimuli on symbolic and identity matching to sample: Evidence for altered instructional processes in pigeon short-term memory
}

\author{
ANGELO SANTI \\ Wilfrid Laurier University, Waterloo, Ontario, Canada \\ and \\ JO TOMBAUGH and T. N. TOMBAUGH \\ Carleton University, Ottawa, Ontario, Canada
}

\begin{abstract}
Two experiments were performed to determine the effects of omitting the comparison stimuli in a matching-to-sample task. In Experiment 1, birds were trained initially on both symbolic and identity matching to sample. Comparison stimuli were then omitted following the presentation of a particular sample stimulus, and this decreased the number of sample (observing) responses. The reintroduction of the comparison stimuli on subsequent probe trials revealed that the accuracy of symbolic matching was reduced to chance levels, while identity matching accuracy was significantly below chance. In Experiment 2, a similar procedure was employed; however, observing responses to the comparison-omitted samples were maintained by direct reinforcement (fixed ratio 20). Matching accuracy during probe trials was again at chance levels for symbolic matching but, contrary to Experiment 1, was significantly above chance for identity matching. The differential effects of omitting comparison stimuli on symbolic and identity matching trials in these two experiments were interpreted within a framework which assumes that instructional processes are altered by comparison-omission procedures.
\end{abstract}

Delayed matching to sample has been extensively employed as a paradigm for studying short-term memory and information processing in pigeons. In this paradigm, the correct response is to peck the comparison stimulus that is the same as the previously presented sample stimulus. The sample stimulus is displayed alone at the beginning of each trial, and the presentation of the comparison stimuli is contingent on responding to the sample one or more times. These sample responses are called observing responses. Considerable evidence indicates that matching accuracy is directly related to the number of observing responses required (Roberts, 1972; Sacks, Kamil, \& Mack, 1972), the occurrence of samplespecific behaviors (Urcuioli \& Honig, 1980; Zentall, Hogan, Howard, \& Moore, 1978), and the duration of sample presentation (Grant, 1976). Furthermore,

This research was supported by Grant A9801 from the National Research Council of Canada to Jo Tombaugh, Grant A7074 from the Natural Sciences and Engineering Research Council of Canada to Tom N. Tombaugh, and by Grant A6378 from the Natural Sciences and Engineering Research Council of Canada to Angelo Santi. Requests for reprints should be sent to Angelo Santi, Department of Psychology, Wilfrid Laurier University, Waterloo, Ontario N2L 3C5, Canada.
Maki, Gillund, Hauge, and Siders (1977) have demonstrated that observing responses can be extinguished by omitting the comparison stimuli for that particular sample over a number of sessions. On subsequent test trials, when comparison stimuli were reintroduced for the sample, matching accuracy decreased to chance levels.

A two-stage model of sample processing was proposed by Maki et al. (1977) to explain these results. In the first stage, the pigeon merely recognizes the sample and decides whether to continue observing (e.g., makes an absolute judgment). In the second stage, observing responses provide the basis for subsequent matching behavior by allowing for additional processing. That is, the information crucial to a correct matching response is not available unless a bird continues to observe a sample after its initial presentation. This interpretation is similar to that derived from trace strength theory (Roberts, 1972), which states that, while information crucial to a correct matching response is available upon initial presentation of the sample and is strengthened by continued observing of the sample, it rapidly decays in the absence of observing responses.

Both hypotheses assume that losses in matching accuracy are directly caused by extinction of the ob- 
serving response rather than by other factors such as forgetting, avoidance of the less frequently reinforced comparison, or an altered contingency between sample and comparison stimuli. The forgetting hypothesis proposed that omission of comparison stimuli eliminates the opportunity to make appropriate matching responses to particular sample stimuli. This, in turn, facilitates forgetting those matching rules specifically related to those sample stimuli. This explanation may be rejected on the basis of the Maki et al. (1977) finding that simply omitting both comparison stimuli and their samples, instead of omitting only the comparison, does not decrease matching accuracy. In addition, Maki et al. also provide evidence that extinction of observing responses to a particular sample stimulus (e.g., green) does not produce a response bias away from the less frequently reinforced comparison stimulus (i.e., the omitted green comparison). This hypothesis was ruled out by the fact that when the same stimulus was presented as the correct comparison in symbolic matching, no response biases were observed, indicating that reduced accuracy was found only when the sample used with the omitted comparison was presented. However, their demonstration that the effects could not be attributed to an altered sample-comparison contingency influencing how the treated samples were encoded was less convincing. They reasoned that if an altered sample-comparison contingency had produced their results, then matching accuracy should also deteriorate following treatment conditions in which comparison stimuli were omitted but the number of observing responses was maintained at a high level. Failure to observe decreased matching accuracy would support the assumption that the original effect was due to extinction of the observing responses. They elected to ensure observing behavior by using food presentation as a sample stimulus (e.g., following a food sample, peck green; following no food, peck red) and reported that performance did not decrease on test trials. They attributed this immunity to the continued observing (i.e., eating) of food samples even though the comparison stimuli had been omitted. However, the use of a biologically significant event such as food for the sample calls into question the appropriateness of generalizing Maki et al.'s conclusion to the original findings. For example, several researchers have suggested that food samples may be more memorable than nonfood samples (Staddon, 1974; Stubbs, Vautin, Reid, \& Delehanty, 1978) and that control exerted by food and nonfood samples over performance may be greater than that exerted by color or form stimuli (Wilkie, 1978).

The present experiments further investigated the role of altered sample-comparison contingencies by using standard visual stimuli (i.e., colors or line tilts) as samples and directly reinforcing observing re- sponses by inserting response-contingent food between the termination of all sample stimuli and presentation of the comparison stimuli. Prior to the implementation of this procedure, the original findings of Maki et al. (1977) were replicated using withinsubject and within-experiment procedures to control for the effects of both amount of forgetting and frequency of reinforcement associated with comparison stimuli.

\section{EXPERIMENT 1}

The original conclusion of Maki et al. (1977) was based on a series of experiments, each successively designed to control for such factors as forgetting, altered sample-comparison contingency, and amount of matching experience. Moreover, in each study only one of the four sample stimuli was selected for treatment, which, as the authors pointed out, produced an unbalanced design in which one of the comparison stimuli was correct twice as often as its corresponding comparison. This necessitated a series of detailed comparisons between specific subsets of sample-comparison combinations in order to exclude the possibility that the results were due to the birds' learning to avoid the less frequently reinforced comparison stimuli. The present experiment eliminated this potential source of response bias by employing a balanced within-subject design in which comparison stimuli were omitted for two of the four original sample stimuli. For one sample, only the comparison stimuli were omitted, while, for the other, both sample and comparison stimuli were omitted. This resulted in other comparison stimuli having an equal probability of being correct on the remaining trials within a session. Additionally, the stimulus that was omitted entirely provided a within-subject control procedure for forgetting. Data from this condition should indicate the extent to which the lack of opportunity to match sample and comparison stimuli contributed to the results. Previously, Maki et al. (1977) indicated that forgetting through "disuse" of the comparison was not a critical factor. However, since this conclusion was based on data obtained from birds with differing degrees of experimental training, it is difficult to assess the relative influence that forgetting may have had on performance, and the withinsubject control provides a more sensitive assessment.

\section{Method}

Subjects. Eight experimentally naive White Carneaux pigeons, with unrestricted access to water and grit in their home cages, were maintained at $\mathbf{8 0 \%}$ of their free-feeding weights throughout the experiment.

Apparatus. Four Lehigh Valley pigeon chambers (Model No. 1519) were used. Each chamber was enclosed in a plywood box insulated with acoustical ceiling tile. Masking noise and ventilation were provided by two 100-CFM Dayton blowers located on the left side wall and back wall, respectively, of each appara- 
tus and holding box. The three translucent (Perspex) response keys were all $2.54 \mathrm{~cm}$ in diameter. The stimuli used (red, green, and a white line with an orientation of 0 or $90 \mathrm{deg}$ from horizontal) could be projected on the back of the translucent keys by an In-Line Digital Unit. Directly below the center key was a 5.08-cm square opening that provided access to a hopper filled with mixed grain. General illumination was provided by a houselight located above the center response key. All experimental contingencies and response measures were programmed and recorded by a PDP-8I digital computer located in a separate room.

Procedure. All birds were hopper trained and autoshaped to peck the keys. Following this preliminary training, birds were given zero-delay matching-to-sample training. Each trial began with a white light projected on the center key. A single peck on the key resulted in one of four samples (red or green or a vertical or horizontal line) replacing the white light. After 10 additional pecks [fixed ratio-(FR) 10], the center key darkened and the side keys were illuminated by red and green. A peck at either side key darkened both keys for $12 \mathrm{sec}$ and illuminated the houselight. If the peck was at the correct comparison stimulus, then it was followed by 3-sec access to grain. The red side key was correct following samples of red or vertical, and the green side key was correct following samples of green or horizontal. Each session consisted of 96 trials (12 randomized blocks of the 8 different stimulus configurations). Each sample appeared twice in each block, and the red and green comparisons were counterbalanced over the left and right keys. This phase was in effect for 40 days, and on the last 3 days matching-to-sample accuracy ranged from $81 \%$ to $95 \%$ correct.

During the treatment phase, three types of trials occurred: (1) configuration-unaltered, (2) comparison-omitted, and (3) sample-omitted. On configuration-unaltered trials, two of the original four sample stimuli continued to be presented as they were in the training phase. On comparison-omitted trials, one of the four sample stimuli was presented for a fixed time of $5 \mathrm{sec}$, but no comparison stimuli were presented. For these trials, termination of the sample was followed by an intertrial interval (ITI). On sample-omitted trials, the remaining sample stimulus was omitted along with the comparison stimuli. For these trials, a single peck to the white light, which began each trial, darkened the center key and initiated an ITI. The stimulus configurations assigned to the various trial types for each bird are presented in Table 1. This treatment phase continued for 26 sessions for all birds, and rates of pecking the comparison-omitted sample did not

Table 1

Stimulus Combinations for Three Types of Trial in Experiments 1 and 2

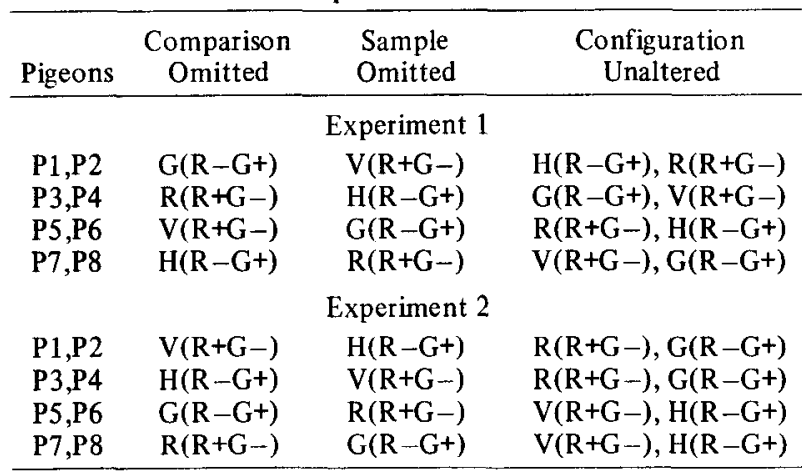

Note-Stimulus abbreviations are red $(R)$, green $(G)$, vertical line $(V)$, and horizontal line $(H)$. For each matching trial, the sample stimulus is indicated first, followed by the comparison stimuli within parentheses. The correct comparison is designated by + and the incorrect by -. Both comparisons were counterbalanced with respect to position. exceed .5 responses $/ \mathrm{sec}$ at the end of this phase and are similar to those reported by Maki et al. (1977).

During the test phase, test trials were interspersed among the regular trials. Sessions consisted of 10 blocks of 8 trials. The first and last block of a session were identical to those during the treatment phase. Each of the remaining blocks contained six trials identical to those in the treatment phase and two accuracy test trials. During the accuracy test trials, sample stimuli were presented for $5 \mathrm{sec}$ and were followed by the appropriate comparison stimuli and reinforcement for correct responding. The order of presentation of the sample stimuli during the 16 daily test trials was randomized. Ten test sessions were conducted.

\section{Results}

The .05 level of significance was adopted for all of the statistical tests reported. The data presented in Table 2 include the mean observing response rate, the mean percent correct matching, and the mean comparison latency obtained during test sessions. These data were averaged over the 10 test sessions and are presented as a function of treatment conditions and groups. The three types of treatment conditions were (1) configuration-unaltered, (2) comparison-omitted, and (3) sample-omitted. The group variable indicated whether the comparisonomitted trial constituted an identity matching trial (i.e., sample and correct comparison from the same dimension) or a symbolic matching trial (i.e., sample and correct comparison from a different dimension). During test sessions, the observing response rate on comparison-omitted trials was very low and a statistically significant effect of treatment conditions was obtained $[\mathrm{F}(2,12)=90.42]$. In addition, a significant treatment conditions $\times$ group interaction occurred $[F(2,12)=4.06]$. A simple main effects analysis revealed that the effect of treatment conditions was statistically significant for both the identity matching $[F(2,12)=30.40]$ and symbolic matching $[F(2,12)=$ 64.08] groups. Newman-Keuls tests indicated that, for both identity and symbolic matching groups, the mean observing response rate for the comparisonomitted condition was significantly lower than the rate for either the sample-omitted or configurationunaltered conditions. The significant interaction was a result of the higher mean rate of observing responses in the sample-omitted condition for the symbolic group than for the identity group $[F(1,12)=6.91]$. The mean rate of observing response did not differ as a function of groups in either of the two remaining treatment conditions (both Fs $<2$ ).

Mean percent correct matching performance is also shown in Table 2. Matching accuracy was substantially lower on comparison-omitted trials for both identity matching and symbolic matching groups. An analysis of variance revealed a statistically significant effect of treatment conditions $[F(2,12)=$ 47.01 ], but the treatment condition $\times$ group interaction was not significant $(\mathrm{F}<2)$. A Newman-Keuls test indicated that matching accuracy was significantly lower on comparison-omitted trials than on 
Table 2

Mean Observing Response Rate, Mean Percent Correct Matching, and Mean Comparison Latency as a Function of Treatment Conditions and Groups in Experiment 1

\begin{tabular}{cccc}
\hline & \multicolumn{3}{c}{ Treatment Condition } \\
\cline { 2 - 3 } Group & $\begin{array}{c}\text { Configuration } \\
\text { Unaltered }\end{array}$ & $\begin{array}{c}\text { Comparison } \\
\text { Omitted }\end{array}$ & $\begin{array}{r}\text { Sample } \\
\text { Omitted }\end{array}$ \\
\hline Identity & \multicolumn{2}{c}{ Mean } & Observing Response Rate \\
Symbolic & 3.45 & .96 & 2.89 \\
& 3.80 & .50 & 3.77 \\
Identity & \multicolumn{3}{c}{ Mean Percent Correct Matching } \\
Symbolic & 86.0 & 37.0 & 67.5 \\
& 97.0 & 58.0 & 92.3 \\
Identity & \multicolumn{2}{c}{ Mean Comparison Latency } \\
Symbolic & .78 & 1.40 & .94 \\
\hline
\end{tabular}

either sample-omitted or configuration-unaltered trials. Accuracy on sample-omitted trials was also significantly lower than on configuration-unaltered trials. A statistically significant group effect was also obtained due to an overall lower level of matching accuracy by the identity matching group than by the symbolic matching group $[\mathrm{F}(1,6)=10.25]$. Additional analyses revealed that, in the comparisonomitted condition, the accuracy of the identity group was significantly below chance $[t(3)=-2.48]$, while the accuracy for the symbolic group did not significantly differ from chance $[t(3)=2.10, p>.05]$.

An analysis of variance of the mean comparison latency data was also undertaken, but the results failed to reveal statistically significant effects of either treatment conditions $[F(2,12)=2.88, p>.05]$ or groups $(F<1)$, or a treatment conditions $\times$ groups interaction $(F<1)$.

\section{Discussion}

The current results replicated the original finding reported by Maki et al. (1977) that comparisonomission procedures extinguish observing responses to treated samples and subsequently disrupt matching accuracy. The extent to which this loss in matching accuracy is directly caused by observing response extinction as opposed to forgetting the rules governing matching for comparison-omitted samples can be directly assessed by examining performance on sampleomitted trials. While the sample-omitted condition did show a significant decrease in accuracy relative to the comparison-unaltered condition, it nevertheless did result in significantly higher levels of accuracy than the comparison-omitted condition. Hence, while forgetting is a factor, it cannot account for all of the decrease in accuracy observed on comparisonomitted trials.

The data presented on comparison latencies help discard the additional hypothesis that the comparisonomission effects are essentially the result of inserting a delay between samples and comparisons. There were no significant differences obtained in comparison latencies, although the effect of treatment conditions did approach significance. Even if significant differences in comparison latencies had been obtained, data from other studies suggest that they could not account for the comparison-omission effect. For example, Maki, Olson, and Rego (1981) have shown that, in a directed forgetting procedure, in which brief postsample cues signal whether comparisons will be omitted or presented, incrementing the delay interval by an amount of time comparable to the increase in choice latencies has no effect on matching accuracy.

It is also evident that the loss in matching accuracy cannot be viewed as a direct consequence of observing response extinction, as is suggested by the processing hypothesis advanced by Maki et al. (1977). One implication of this hypothesis is that, following observing response extinction, information about the sample should not be available when the comparison stimuli are presented. The finding that identity matching was significantly below chance during testing is clearly inconsistent with this expectation, since this could have occurred only if the birds were responding on the basis of sample characteristics. Apparently, sample information is available and used in spite of a considerable reduction in observing responses.

The present results can be interpreted in terms of the comparison-omission procedure altering instructional processes (Honig, 1978; Roitblat, 1980). Assume that sample stimuli generate specific instructions in short-term memory on each trial. As the sample changes from trial to trial, so do the instructions. For comparison-omitted trials in Experiment 1, it is assumed that the sample generates two instructions: (1) do not peck that specific stimulus (i.e., red or green, vertical line or horizontal line) and (2) do not encode and/or rehearse instructions for choice behavior. When comparison stimuli are reintroduced during testing for comparison-omitted trials, the normal instruction for choice behavior is not available, but there is an instruction to refrain from pecking that specific stimulus. If the comparison-omitted trial consisted of identity matching, this would bias the birds to select the incorrect comparison stimulus and produce the below-chance levels of accuracy reported in Experiment 1. However, if the comparisonomitted trial consisted of symbolic matching, no bias would occur since comparison stimuli are on a different dimension. Hence, as reported, symbolic matching accuracy would not significantly differ from chance during testing.

\section{EXPERIMENT 2}

In Experiment 2, the effects of omitting comparison stimuli were investigated when observing responses were directly reinforced. This procedure was 
introduced in order to investigate the degree to which the omission of the comparison stimulus per se was responsible for the results originally reported by Maki et al. (1977) and subsequently replicated in Experiment 1 . As noted earlier, Maki et al. attempted to guarantee the occurrence of observing responses on comparison-omitted trials by using food as the sample stimulus (e.g., if food, then peck green; if no food, then peck red). In the present experiment, observing responses to a visual sample were maintained by directly reinforcing completion of the response requirement prior to the omission of the comparison stimuli. According to the processing hypothesis advanced by Maki et al. (1977), the continued emission of observing responses during the comparison-omission treatment should result in high levels of matching accuracy when the comparison stimuli are subsequently reinstated. Moreover, this should occur regardless of whether the trials involve identity matching or symbolic matching. However, according to the interpretation advanced in Experiment 1, the altered sample-comparison contingency in Experiment 2 should generate a new instruction in short-term memory. Because of the direct reinforcement of observing responses on comparison-omitted trials, it is assumed that the sample would generate the following instructions: (1) peck that specific stimulus and (2) do not encode and/or rehearse instructions for choice behavior. On comparison-omitted test trials in Experiment 2 , the usual instruction for choice behavior would not be available, but there would be an instruction to peck the specific stimulus presented as the sample on that trial. Hence, in Experiment 2, identity matching accuracy should be significantly greater than symbolic matching accuracy and also significantly greater than chance.

\section{Method}

Subjects and Apparatus. The same subjects and apparatus as previously described were employed.

Procedure. All birds were retrained on the original delayed matching-to-sample task, except that $1.5-\mathrm{sec}$ access to mixed grain was provided following completion of the sample response requirement (FR 10). Following the 1.5-sec interval, the comparison stimuli were presented and choice behavior was appropriately reinforced. Percent correct responding ranged from $65 \%$ to $85 \%$ after 52 days. In an effort to increase accuracy to a level comparable to that observed in Experiment 1, the sample response requirement was increased to FR 20. Accuracy increased on the following 6 days until, on the last day of training, it ranged from $83 \%$ to $96 \%$. The FR 20 continued in effect during the rest of the experiment.

For each subject, the sample to be used in the sample-omission condition was that used in the configuration-unaltered condition in Experiment 1, while the sample used in the comparison-omitted condition had been employed previously in the sample-omitted condition (see Table 1, Experiment 2). Otherwise, the treatment conditions were identical to those described in Experiment 1 except for two important modifications. First, the 1.5-sec access to mixed grain following completion of the FR requirement continued to be programmed on the comparison-omitted and configuration-unaltered trials. Second, in order to insure that
}

birds in these two conditions emitted the same number of observing responses per trial, the FR-20 schedule remained in effect for sample responses, and the fixed time procedure previously described in Experiment 1 was not used. Twenty-seven sessions were employed.

The same testing techniques employed in Experiment 1 were used, except the 1.5-sec access to mixed grain continued to be interpolated between the termination of the sample and presentation of the comparison stimulus. Ten sessions were given.

\section{Results}

The .05 level of significance was adopted for all of the statistical tests reported. The data presented in Table 3 include the mean time $(\mathrm{sec})$ to complete the FR 20, the mean percent correct matching, and the mean comparison latency averaged over the 10 test sessions as a function of treatment conditions and groups. An analysis of variance performed on the mean time to complete the FR 20 revealed a significant treatment effect $[F(2,12)=5.89]$. A NewmanKeuls test indicated that the time required was significantly greater on comparison-omitted trials than on either configuration-unaltered or sample-omitted trials. The configuration-unaltered and sample-omitted conditions did not significantly differ. Neither the effect of groups $[F(1,6)=3.53, p>.10]$ nor the treatment conditions $\times$ group interaction $[F(2,12)=2.78$, $p>.10]$ was statistically significant.

An analysis of variance of the mean percent correct matching data indicated a significant effect of group $[F(1,6)=10.53]$ and treatment conditions $[\mathrm{F}(2,12)=9.80]$, and a treatment conditions $\times$ group interaction $[F(2,12)=7.58]$. A simple main effects analysis revealed a significant effect of treatment conditions for the symbolic matching group $[F(2,12)$ $=17.11]$, but not for the identity matching group $(F<1)$. A Newman-Keuls test performed on the accuracy data for the symbolic matching group indicated that matching accuracy was greater on

Table 3

Mean Time to Complete the FR Requirement, Mean Percent Correct Matching, and Mean Comparison Latency as a Function of Treatment Conditions and Groups in Experiment 2

\begin{tabular}{cccc}
\hline & \multicolumn{3}{c}{ Treatment Condition } \\
\cline { 2 - 3 } Group & $\begin{array}{c}\text { Configuration } \\
\text { Unaltered }\end{array}$ & $\begin{array}{c}\text { Comparison } \\
\text { Omitted }\end{array}$ & $\begin{array}{c}\text { Sample } \\
\text { Omitted }\end{array}$ \\
\hline Mean Time (in Seconds) to Complete \\
Identity & \multicolumn{3}{c}{ the FR Requirement } \\
Symbolic & 5.51 & 5.16 & 4.59 \\
& 5.40 & 9.06 & 6.41 \\
Identity & \multicolumn{4}{c}{ Mean Percent Correct Matching } \\
Symbolic & 87.8 & 84.4 & 89.4 \\
& 95.6 & 56.9 & 69.4 \\
Identity & \multicolumn{3}{c}{ Mean Comparison Latency } \\
Symbolic & 1.01 & 1.09 & 1.09 \\
\hline
\end{tabular}


configuration-unaltered trials than on either comparison-omitted or sample-omitted trials. Accuracy on comparison-omitted and sample-omitted trials did not differ significantly. The simple main effects analysis also indicated that the identity matching group had a higher level of matching accuracy than the symbolic matching group on comparisonomitted trials $[F(1,12)=16.56]$ and on sample-omitted trials $[F(1,12)=8.76]$, but not on configurationunaltered trials $[F(1,12)=1.34, p>.25]$. An additional analysis of the accuracy data on comparisonomitted trials showed that the identity matching group's accuracy was significantly greater than chance $[t(3)=8.88]$, while the symbolic matching group's accuracy did not differ significantly from chance $(t<1.0)$.

An analysis of comparison latencies was also undertaken. Only the effect of treatment conditions was significant $[F(2,12)=7.34]$. A Newman-Keuls test showed that comparison latencies in the configuration-unaltered condition were significantly lower than in either the comparison-omitted or the sample-omitted condition.

\section{Discussion}

The present results show that when observing responses are directly reinforced, omission of the comparison stimuli had different effects on accuracy of identity and symbolic matching. This relationship, observed even though equivalent numbers of observing responses were maintained to samples on both types of trials, is inconsistent with the assumption made by Maki et al. (1977) that losses in matching accuracy were directly caused by observing response extinction. It would appear that maintaining observing responses to visual stimuli in a comparisonomission procedure produces results different from those they obtained by using food vs. no-food as sample stimuli. One possible factor contributing to these differential effects is the opportunity for control by sample-specific behaviors (Urcuioli \& Honig, 1980). Using food vs. no-food as samples would generate markedly different sample-specific behaviors, and these sample-specific behaviors could eventually control choice responses. The probability of differential sample-specific behaviors would not be as great with visual samples as with food vs. no-food samples. The current data are also inconsistent with a memory hypothesis (Roberts, 1972), which assumes that the strength of the initial representation of a sample is maintained by observing responses. This hypothesis also predicts equivalent performance across treatment conditions since the same number of observing responses were made. In addition, since longer times were required to complete the response requirement on comparison-omitted trials, the memory hypothesis would incorrectly predict higher rather than lower levels of accuracy on these trials since greater expo- sure to the sample stimulus would have occurred, thereby producing a stronger stimulus trace (Grant, 1976).

The present data, on the other hand, are consistent with the hypothesis developed in Experiment 1 that the comparison-omission procedure alters instructional processes in short-term memory. In Experiment 2, it was predicted that as a result of direct reinforcement of observing responses, sample stimuli for which comparisons had been omitted would generate instructions to peck at that specific sample and not to encode and/or rehearse instructions for choice behavior. Consequently, when comparisons were reinstated following these samples during testing, the only active instruction on these trials was to peck at the specific stimulus presented as the sample. While this artificially produced a level of identity matching accuracy significantly above chance, it resulted in a level of symbolic matching accuracy that was equivalent to chance performance.

However, there are several potentially confounding variables that should be considered. One set of variables pertains to the possible role of accumulated experience resulting from the use of the same subjects in both experiments and the change from an FR 10 to an FR 20 in Experiment 2. First, it should be noted that the same group of birds behaved very differently in the comparison-omitted conditions of the two experiments. One group (P1-P4) was significantly below chance in Experiment 1 (i.e., identity) but at chance in Experiment 2 (i.e., symbolic); in contrast, the other group (P5-P8) performed at chance levels in Experiment 1 (i.e., symbolic) and was well above chance in Experiment 2 (i.e., identity). The fact that both groups of birds performed at a greater level of accuracy in the comparison-omitted condition of Experiment 2 relative to Experiment 1 could possibly be attributed to accumulated experience. However, results for the sample-omitted condition are not consistent with this hypothesis; that is, one group of birds (P1-P4) had accuracy levels of $67.5 \%$ in Experiment 1 (i.e., identity) and 69.4\% in Experiment 2 (i.e., symbolic), while the other group of birds (P5-P8) had accuracy levels of $92.3 \%$ in Experiment 1 (i.e., symbolic) and $89.4 \%$ in Experiment 2 (i.e., identity). Hence, performance on sample-omitted trials remained constant from Experiment 1 to Experiment 2 despite the change in FRs, the insertion of a reinforcer after the FR in Experiment 2, and accumulated experience in the study. The differences in accuracy on sample-omitted trials between the two groups of birds is most likely the result of the sample-omitted condition's being administered on a symbolic matching trial for one group (P1-P4) but on an identity matching trial for the other group (P5-P8). Omitting a symbolic matching trial entirely over several sessions apparently produces a performance decrement, whereas omitting an identity matching trial entirely 
over the same number of sessions does not. It is likely for two reasons that this would be the case. First, the omitted sample stimulus from a symbolic matching trial (i.e., vertical or horizontal line) was entirely absent from the experiment for the duration of the treatment phase, while the omitted sample stimulus from an identity matching trial (i.e., red or green) continues to be presented as a comparison stimulus. Second, acquisition of matching performance is known to be more difficult with line tilt stimuli than with colors (Carter \& Werner, 1978), and this raises the possibility that forgetting might also occur more rapidly with these stimuli.

One final variable that conceivably might confound the symbolic-identity differences observed during the comparison-omitted condition of both experiments is the assignment of sample stimuli to treatment conditions; that is, in Experiment 1 the samples for the comparison-omitted and sampleomitted conditions were drawn from different dimensions, while in Experiment 2 the samples in these two conditions were drawn from the same dimension. Clearly, whether the dimensions are similar or different has no effect on performance in the sampleomitted condition of the two experiments since, as pointed out above, the accuracy levels remain stable across experiments. In addition, it can be easily shown that this difference also is not terribly important in the comparison-omitted condition. Consider what occurred when a symbolic matching trial was selected for comparison-omission. Performance decreased to chance levels in the comparison-omitted condition of both experiments regardless of whether the sampleomitted trial involved a sample from the same dimension (Experiment 2) or from a different dimension (Experiment 1). In addition, when a symbolic trial was selected for comparison-omission, consistent results were obtained across experiments regardless of whether Birds P5-P8 experienced this condition (as in Experiment 1) or Birds P1-P4 experienced it (as in Experiment 2). The crucial difference between the two experiments rests with the data obtained when an identity matching trial was selected for comparisonomission. If responding to the sample was not reinforced (as in Experiment 1), then accuracy was sig- nificantly below chance, but, if responding to the sample was reinforced (as in Experiment 2), then accuracy was significantly above chance. The most parsimonious interpretation of these results would appear to be that comparison-omission procedures alter instructional processes in pigeon short-term memory.

\section{REFERENCES}

Carter, D. E., \& Werner, T. J. Complex learning and information processing by pigeons: A critical analysis. Journal of the Experimental Analysis of Behavior, 1978, 29, 565-601.

Grant, D. S. Effect of sample presentation time on long-delay matching in the pigeon. Learning and Motivation, 1976, 7, 580-590.

HoniG, W. K. Studies of working memory in the pigeon. In S. H. Hulse, H. Fowler, \& W. K. Honig (Eds.), Cognitive processes in animal behavior. Hillsdale, N.J: Erlbaum, 1978.

Maki, W. S., Gillund, G., Hauge, G., \& Siders, W. A. Matching to sample after extinction of observing responses. Journal of Experimental Psychology: Animal Behavior Processes, 1977, 3, 285-296.

Makı, W. S., Olson, D., \& Rego, S. Directed forgetting in pigeons: Analysis of cue functions. Animal Learning \& Behavior, 1981, 9, 189-195.

RoвERTS, W. A. Short-term memory in the pigeon: Effects of repetition and spacing. Journal of Experimental Psychology, 1972, 94, 74-83.

Roitblat, M. L. Codes and coding processes in pigeon short-term memory. Animal Learning \& Behavior, 1980, 8, 341-351.

Sacks, R. A., Kamil, A. C., \& Mack, R. The effects of fixedratio sample requirements on matching to sample in the pigeon. Psychonomic Science, 1972, 26, 291-293.

Staddon, J. E. R. Temporal control, attention, and memory. Psychological Review, 1974, 81, 375-391.

Stubis, D. A., Vautin, S. J., Reid, H. M., \& Delehanty, D. L. Discrimination functions of schedule stimuli and memory: A combination of schedule and choice procedures. Journal of the Experimental Analysis of Behavior, 1978, 29, 167-180.

URcuioli, P. J., \& Honta, W. K. Control of choice in conditional discriminations by sample-specific behaviors. Journal of Experimental Psychology: Animal Behavior Processes, 1980, 6, 251-277.

WiLkIE, D. M. Delayed symbolic matching to sample in the pigeon. Psychological Record, 1978, 28, 463-469.

Zentall, T. R., Hogan, D. E., Howard, M. M., \& Moore, B. S. Delayed matching in the pigeon: Effect on performance of sample-specific observing responses and differential delay behavior. Learning and Motivation, 1978, 9, 202-218.

(Manuscript received August 3, 1981;

revision accepted for publication December 22, 1981.) 